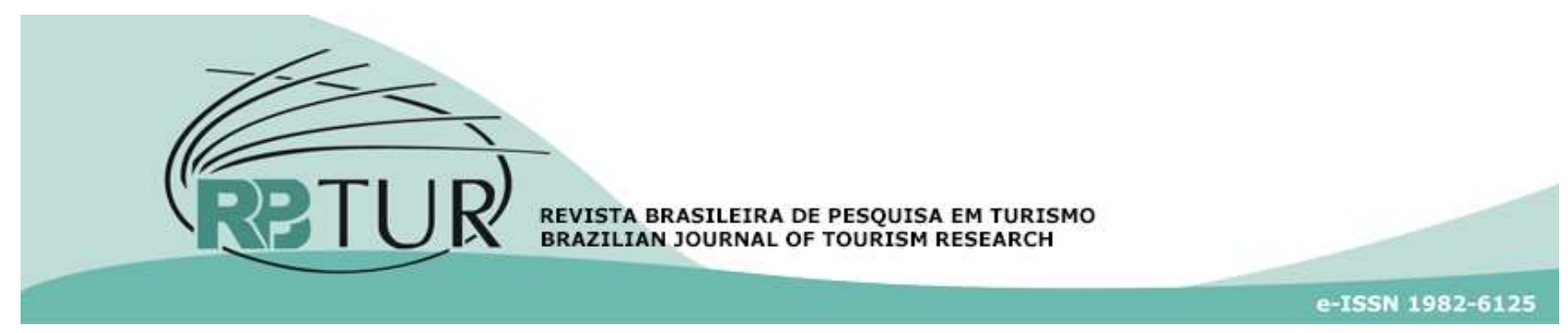

Artigo

DOI: $\underline{\text { http://dx.doi.org/10.7784/rbtur.v12i2.1419 }}$

\title{
Caracterização das atividades turísticas nos municípios brasileiros em 2015
}

\section{Characteristics of tourism activities in brazilian municipalities in 2015}

\section{Caracterización de las actividades turísticas en los municipios brasileños en 2015}

\author{
Fernanda Rodrigues dos Santos ${ }^{1}$ \\ Luiz Carlos de Santana Ribeiro ${ }^{2}$ \\ Emanuel Jhonata Gomes da Silveira ${ }^{3}$
}

Resumo: O objetivo deste artigo é caracterizar o setor turístico nos municípios brasileiros no ano de 2015. Para isso, utilizou-se a análise de cluster considerando o número de empregos, o número de estabelecimentos para cada 100.000 habitantes, a renda média, o percentual de trabalhadores com o ensino superior completo e o grau de especialização das atividades turísticas. Os principais resultados indicam a formação de seis clusters. Três deles, com características distintas, podem ser considerados clusters turísticos, uma vez que são constituídos por cidades especializadas em turismo com apelo em diferentes segmentos. Por outro lado, em $90,6 \%$ dos municípios brasileiros (clusters 2 e 3 ) pode-se considerar a atividade turística é incipiente.

Palavras-chave: Atividade turística. Desenvolvimento regional. Cluster. Municípios brasileiros.

Abstract: This paper aims to carry out an analysis of the tourism sector in Brazilian municipalities in 2015. To do so, the cluster analysis was used considering the number of jobs, the number of establishments per 100,000 inhabitants, the average income, the percentage of workers with complete higher education and the specialization degree regarding to the tourism activities. The main results indicate the formation of six clusters. Three of

${ }^{1}$ Graduada em Economia, Universidade Federal de Sergipe. Todos os autores contribuíram em todas as etapas da pesquisa.

2 Professor Assistente do Departamento de Economia, Universidade Federal de Sergipe. Bolsista do CNPq e Coordenador do Laboratório de Economia Aplicada e Desenvolvimento Regional- LEADER. Todos os autores contribuíram em todas as etapas da pesquisa.

3 Mestrando em Economia, Universidade Federal de Sergipe e pesquisador do LEADER. Todos os autores contribuíram em todas as etapas da pesquisa.

Artigo recebido em: 13/02/2018. Artigo aceito em: 27/03/2018.

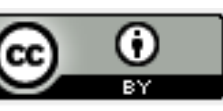


them, with distinct characteristics, can be considered tourist clusters, since they are constituted by cities specialized in tourism with appeal in different segments. On the other hand, $90.6 \%$ of the Brazilian municipalities (clusters 2 and 3 ) can be consider that the tourism activity is incipient.

Keywords: Touristic activity. Regional development. Cluster. Brazilian municipalities.

Resumen: El objetivo de este artículo es caracterizar el sector turístico en los municipios brasileños en el año 2015. Para ello, se utilizó el análisis de cluster considerando el número de empleos, el número de establecimientos por cada 100.000 habitantes, el ingreso promedio, el porcentaje de trabajadores con la enseñanza superior completa y el grado de especialización de las actividades del turismo. Los principales resultados indican la formación de seis clusters. Tres de ellos, con características distintas, pueden ser considerados clusters turísticos, ya que están constituidos por ciudades especializadas en turismo con apelación en diferentes segmentos. Por otro lado, en el $90,6 \%$ de los municipios brasileños (clusters 2 y 3 ) se puede considerar la actividad turística es incipiente.

Palabras clave: Actividad turística. Desarrollo regional. Cluster. Municipios brasileños.

\section{INTRODUÇÃO}

O intenso crescimento do setor turístico ao longo das últimas décadas fez com que o mesmo passasse a ser visto como estratégia para se atingir o desenvolvimento econômico (Ribeiro, Lopes, Montenegro, \& Andrade, 2017a; Ribeiro, Silva, Andrade, \& Souza, 2017b). Segundo o Conselho Nacional de Viagens e Turismo (WTTC, 2016), de cada onze empregos gerados no mundo em 2015, um estava relacionado com a atividade turística. Não obstante, nesse mesmo ano, o turismo foi responsável por $9,8 \%$ do PIB mundial. Na medida em que a atividade turística estabelece relações diretas e indiretas com outros setores, a renda gerada por ela circula na economia potencializando o fluxo de renda da localidade receptora.

O turismo no Brasil, em 2016, movimentou $\mathrm{R} \$ 530,5$ bilhões entre atividades diretas, indiretas e induzidas. Estima-se que, em 2027, o impacto do turismo represente 9,1\% do PIB brasileiro (Tomé, 2017). Mesmo com esta representatividade econômica, ainda há deficiências relevantes no que remete à construção e divulgação de informa- ções estatísticas sobre o turismo no país como a inexistência de uma Conta Satélite.

Há de se reconhecer, contudo, o esforço recente do setor público no sentido de elaborar novas informações estatísticas como, por exemplo, dados de informalidade das Atividades Características do Turismo (ACTs). De acordo com informações do Instituto de Pesquisa Econômica Aplicada (IPEA, 2015), em 2013, o número de ocupações ligadas às ACTs no Brasil era de aproximadamente 1.938 milhões, sendo que, deste total, $51 \%$ correspondia ao mercado de trabalho formal e $49 \%$ aos empregos informais. Vale ressaltar que, entre 2012 e 2014, houve redução no percentual de informalidade das ACTs nas regiões brasileiras (ver Anexo $1 \mathrm{~A}$ e 1B). Cultura e Lazer, dentre os setores que compõe as ACTs, foi o que apresentou maior percentual de informalidade no ano de 2014 (64\%), seguido de Alimentação (57\%). Por outro lado, o Transporte Aéreo apresentou o menor percentual, uma média de $5,3 \%$ no período analisado. Percebe-se, portanto, uma heterogeneidade no grau de formalidade entre as ACTs.

Com a finalidade de estudar o setor 
turístico brasileiro pela ótica da oferta, o problema a ser tratado no presente artigo é analisar se esta atividade, a nível municipal, é mais desenvolvida e especializada nas regiões mais ricas do país, Sul e Sudeste. Ainda que o grau de informalidade nas ACTs seja relevante, como discutido anteriormente, este artigo considera apenas o mercado de trabaIho formal em razão da ausência de dados municipais recentes que levam em conta a informalidade.

Diante disso, o objetivo deste artigo é caracterizar o setor turístico dos 5.565 municípios brasileiros no ano de 2015. Esta análise é conduzida a partir da identificação de grupos de municípios de acordo com o número de estabelecimentos, renda média, grau de escolaridade, emprego e nível de especialização dos serviços turísticos. Todos esses dados foram obtidos na Relação Anual de Informações Sociais (RAIS) do Ministério do Trabalho e Emprego. Com a finalidade de agrupar os municípios brasileiros de acordo com as características dos seus serviços turísticos, optou-se pela utilização da análise de cluster. Por meio desse método, é possível agrupar variáveis pré-estabelecidas em grupos homogêneos (Hair Jr, Anderson, Tathan, \& Black, 2005).

A análise de cluster é amplamente utilizada na literatura. Podem-se destacar estudos nas áreas de turismo (Ribeiro \& Andrade, 2015; Hurtado \& Bonilla, 2012; Moreira Filho, Nascimento, \& Segre, 2010; Lins, 2000); setor cultural (Machado, Simões, \& Diniz, 2013; Ribeiro \& Lopes, 2015), finanças (Massardi \& Abrantes, 2014), entre outros.

Além desta introdução e das considerações finais, o presente artigo está dividido em mais três seções. A primeira seção discute a relação entre turismo, desenvolvimento e políticas públicas. A segunda seção apresenta a análise de cluster, a base de dados utilizada e o tratamento das variáveis. $A$ terceira seção sintetiza os principais resultados e discussões. Por fim, são tecidas as considerações finais e indicadas as referências bibliográficas.

\section{TURISMO, DESENVOLVIMENTO E POLÍ- TICAS PÚBLICAS}

Esta seção aborda a importância da atividade turística para o desenvolvimento local/regional, ou seja, identifica de que forma essa atividade pode contribuir para o desenvolvimento, além de elencar alguns dos efeitos positivos e negativos provocados pela atividade.

O turismo gera efeitos multiplicadores ao estimular outros setores econômicos, o que pode potencializar o desenvolvimento. Isto ocorre porque o turista não demanda apenas produtos turísticos no destino, mas também bens e serviços de outros segmentos. Desse modo, o turismo pode provocar três tipos de impactos econômicos: i) direto; que resulta da geração de empregos provenientes da própria atividade; ii) induzido; causado pelos gastos realizados pelos turistas e iii) indireto; que é o efeito multiplicador na economia provocado por esses gastos ( $\mathrm{Ri}$ beiro \& Lopes, 2015).

Em outras palavras, um gasto inicial decorrente do turismo provoca efeitos múltiplos na economia, pois ele afeta a produção, o emprego, o nível de renda e a receita do governo. Não obstante, a atividade turís- 
tica pode ocasionar aumento real da renda dos moradores locais.

Vale ressaltar que mesmo as localidades que não recebem turistas podem se beneficiar via efeitos de transbordamentos de renda e emprego. Para isso, basta que sejam fornecedoras de produtos demandados pelos turistas como, por exemplo, produtos artesanais e produtos agrícolas. Percebe-se que os reflexos do turismo ultrapassam os limites do destino turístico, uma vez que localidades vizinhas também podem ser beneficiadas (Barbosa, 2005). Nesse quesito, Lazzaretti e Capone (2009) estimaram para a Itália que o crescimento do emprego do turismo nos municípios vizinhos aumenta o emprego local na mesma atividade. Ribeiro et al. (2017a), por outro lado, encontram o efeito inverso para o Brasil, ou seja, o aumento do emprego turístico nos municípios vizinhos reduz o emprego no mesmo setor localmente.

O turismo proporciona uma redistribuição da renda, ou seja, ocorre um fluxo monetário de várias regiões (origem) para o destino turístico. Quando o mesmo tem um baixo nível de desenvolvimento, ele pode utilizar seu potencial turístico, seja ele natural ou cultural, como um mecanismo para atrair investimentos e gerar empregos (Ablas, 1991). Além disso, alguns estudos têm evidenciado o impacto do turismo sobre a desigualdade. Haddad, Porsse e Rabahy (2013) e Ribeiro et al. (2017b), por exemplo, argumentam que a atividade turística no Brasil contribui para a redução das desigualdades regionais.

Anato (2006) ressalta o papel da internet no desenvolvimento da atividade turística. Esta ferramenta possibilitou o surgimento de inúmeras agências de viagens on- line, permitindo ao consumidor ter acesso a informações com relação aos destinos de maneira mais rápida e acessível.

A atividade turística, por outro lado, também pode gerar alguns impactos negativos. Entre eles, merecem destaque: i) aumento de preços no local visitado em virtude do intenso consumo dos turistas; ii) dependência da atividade turística; não é recomendável que países em desenvolvimento utilizem o turismo como principal política de desenvolvimento, pois a demanda turística também está sujeita a influências externas; e iii) impactos culturais e ambientais; o desenvolvimento de atividades turísticas pode culminar na degradação dos recursos ambiental e cultural da comunidade receptora (Lage \& Milone, 1991).

No que remete às políticas do turismo no Brasil, a criação do Ministério do Turismo (MUR) em 2003 mostra a preocupação do governo brasileiro na adoção de medidas que promovam o desenvolvimento deste setor no país. O Programa de Regionalização do Turismo (PRT) constitui-se como a mais recente política pública deste segmento, o qual visa o desenvolvimento do turismo por meio da participação dos residentes no sentido de potencializar os roteiros turísticos da região (Brasil, 2007).

Além do PRT e outras leis e projetos voltados pra a atividade turística, um dos principais programas atualmente é o PRODETUR Nacional, que tem como objetivo fortalecer a Política Nacional de Turismo. Este programa distribui os investimentos em cinco componentes: i) Produto Turístico; ii) Marketing turístico; iii) Fortalecimento institucional; iv) Infraestrutura e serviços básicos; e v) Gestão ambiental (Ribeiro, Andrade \& 
Pereira, 2013).

\section{ANÁLISE DE CLUSTER, BASE DE DADOS E TRATAMENTO DAS VARIÁVEIS}

\subsection{Análise de cluster}

$\mathrm{Na}$ literatura há diversas definições sobre cluster. O conceito mais conhecido, todavia, foi o proposto por Porter (1998), que o definiu a partir de uma perspectiva geográfica, ou seja, uma concentração geográfica de empresas pertencentes a um mesmo setor ou relacionadas a atividades que atuam de maneira complementar. Vale destacar que este autor analisou a formação de clusters no setor industrial.

Este conceito, no entanto, foi adaptado para a área de turismo. Um cluster turístico, para Lins (2000), pode ser definido como um conjunto de atores que engloba as empresas turísticas, o poder público e o poder privado que operam por meio da coordenação, formação de recursos humanos e do planejamento do setor.

De acordo com Moreira Filho et al. (2010) um cluster abrange características específicas de uma região e pode ser caracterizado como sendo horizontal, isto é, quando é composto por empresas que pertencem ao mesmo setor, mas também pode ser vertical, quando engloba empresas que atuam de maneira complementar em diferentes níveis da cadeia produtiva.

Assim como há na literatura diferentes conceitos sobre cluster, também existem diferentes métodos para identificá-los. Hur- tado e Bonilla (2012), por meio da utilização do método de classe latente, classificaram as regiões turísticas da Espanha de acordo com a oferta e a demanda. Os autores utilizaram este método como alternativo à análise de cluster, pelo fato dele apresentar menor critério aleatório para a determinação do número de grupos a serem formados.

Moreira Filho et al. (2010) analisaram a formação de um cluster turístico no bairro de Santa Tereza no Rio de Janeiro ${ }^{4}$ e seu impacto no desenvolvimento local a partir da visão dos atores sociais locais, ou seja, moradores, empresários e representantes da comunidade. Lins (2000), por sua vez, investigou a formação de um cluster turístico na região de Florianópolis a partir das interpelações existentes entre os autores locais.

Um aspecto comum entre todas essas abordagens é o fator geográfico, ou seja, cluster é entendido como um agrupamento delimitado dentro de um espaço. Todavia, a definição de cluster adotada neste artigo é a do método estatístico que leva em consideração as similaridades entre as variáveis dos grupos e desconsidera o fator geográfico. Em outras palavras, é possível ter dois municípios brasileiros de regiões diferentes no mesmo grupo. Ribeiro e Andrade (2015), Ribeiro e Lopes (2015) e Massardi e Abrantes (2014) são exemplos de estudos que aplicaram esta técnica.

Ribeiro e Andrade (2015) caracterizaram os serviços turísticos das mesorregiões brasileiras, em que a renda foi a variável que mais discriminou os grupos. Ribeiro e Lopes (2015), por sua vez, verificaram que as maio-

\footnotetext{
${ }^{4}$ Santa Tereza é um bairro conhecido pela característica histórica e o forte apelo cultural.
} 
res rendas do setor cultural estão no Rio de Janeiro e em São Paulo, municípios constituídos por clusters individuais. Massardi e Abrantes (2014) identificaram que a maioria dos municípios de Minas Gerais depende do Fundo de Participação dos Municípios (FPM).

A análise de cluster ou análise de conglomerado é um procedimento que agrupa as observações de acordo com a sua estrutura original. Porém, diferente de outras técnicas multivariadas, as variáveis são pré-estabelecidas e não estimadas empiricamente (Fávero, Belfiore, Silva \& Chan, 2009). Para Linden (2009), a análise de cluster é uma técnica computacional que busca organizar as observações em grupos de acordo com as características similares. Os grupos formados devem ser internamente homogêneos e externamente heterogêneos.

Como o objetivo desta técnica estatística é agrupar as variáveis que apresentam características semelhantes, é necessário adotar alguma medida de distância. Com efeito, as observações mais próximas entre si serão as mais semelhantes e, portanto, pertencerão ao mesmo agrupamento (Fávero et al., 2009). Para o presente trabalho, foi utilizada a medida de Distância Quadrática Euclidiana, na qual a distância entre as observações ( $i$ e $j$ ) é dada pela soma dos quadrados das diferenças entre i e j para todas as $p$ variáveis, isto é:

$$
d_{i j}^{2}=\sum_{k=1}^{p}\left(x_{i k-} x_{j k}\right)^{2}
$$

Sendo:

$x_{i k}$ : o valor da variável k para a observação i; $x_{j k}$ : o valor da variável k para a observação j.

Todas as variáveis foram padronizadas a fim de eliminar eventuais problemas de escalas. Além disso, dois métodos de agrupamento foram utilizados de forma complementar: os métodos hierárquico e não-hierárquico. $\mathrm{O}$ primeiro define a quantidade de grupos a serem formados. Definida a quantidade de grupos pelo primeiro método, aplicou-se o método não-hierárquico.

Para a definição do número de clusters no método hierárquico, foi utilizado o procedimento de Ward, uma vez que é o mais apropriado quando se utiliza a Distância Quadrática Euclidiana (Hair et al., 2005; Fávero et al., 2009). Para Malhotra (2006, p. 578), este método é um dos mais utilizados e busca-se "minimizar o quadrado da distância euclidiana às médias dos aglomerados".

\subsection{Base de dados e tratamento das variá- veis}

Para agrupar os municípios brasileiros de acordo com as similaridades das atividades turísticas, utilizaram-se as seguintes variáveis coletadas a nível municipal para o ano de 2015: i) número de estabelecimentos turísticos para cada 100.000 habitantes; ii) número de emprego das atividades turísticas em proporção ao número de emprego total; iii) renda média das atividades turísticas; iv) percentual de trabalhadores atuando em atividades turísticas com o ensino superior completo; e v) Quociente Locacional (QL) do turismo.

O QL compara a participação de uma região $j$ em um setor particular $i$ com a participação da mesma região $j$ no total do emprego de todas as regiões (Haddad, 1989). Este trabalho leva em consideração a participação do emprego da atividade turística no total de emprego dos municípios brasileiros 
em comparação com a participação do emprego do turismo no emprego total do Brasil, ou seja:

$$
Q L_{i j}=\frac{E_{i j} / E_{i}}{\mathrm{E}_{\mathrm{j}} / \mathrm{E}}
$$

Em que:

$\mathrm{E}_{i j}$ : emprego do turismo no munícipio $j$;

$E_{i}$ : emprego total do município $j$;

E.j: emprego do turismo no Brasil (região de referência);

E..: emprego total no Brasil.

O QL mede o grau de especialização do turismo em cada município brasileiro. Se $Q L>1$, diz-se que este município é especializado no setor de turismo em relação à região de referência. Diversos trabalhos na literatura têm utilizado o QL como proxy de externalidades de especialização (Glaeser, Kallal, Scheeinkman, \& Shleifer, 1992; Combes, 2000; Aulicino, 2011; Ribeiro et al., 2014; Ribeiro et al., 2017a).

Os dados utilizados foram obtidos na
RAIS, os quais não consideram a informalidade. A única e mais recente base que disponibiliza dados formais e informais em relação ao mercado de trabalho municipal é o Censo Demográfico de 2010 e, portanto, os mesmos não retratariam a atual realidade brasileira. A escassez ou defasagem de dados não podem ser um empecilho para o desenvolvimento de estudos na área do turismo. Com efeito, os dados da RAIS vêm sendo amplamente utilizados na literatura (Garsou, Corderi, Velasco, \& Colombo, 2017; Ribeiro et al., 2017a; Ribeiro \& Andrade, 2015; Ribeiro \& Lopes, 2015).

Para a construção do banco de dados foi utilizado o nível setorial "Classe", do Código Nacional de Atividades Econômicas 2.0 (CNAE 2.0), a qual fornece informações mais desagregadas das atividades. A classificação utilizada aqui para identificar os serviços turísticos foi proposta por Ribeiro e Andrade (2015), em que o setor turístico é composto por 25 atividades econômicas, conforme mostra a Tabela 1.

\section{Classificação Classe CNAE 2.0}

Transporte metroferroviário de passageiros

Transporte rodoviário coletivo de passageiros, com itinerário fixo, municipal e em região metropolitana

Transporte rodoviário coletivo de passageiros, com itinerário fixo, intermunicipal, interestadual e internacional Transporte rodoviário de táxi

Transporte rodoviário coletivo de passageiros, sob regime de fretamento, e outros transportes rodoviários

Transporte turístico, teleférico e similares

Transporte marítimo de cabotagem

Transporte por navegação interior de passageiros em linhas regulares

Transporte aéreo de passageiros regular

Transporte aéreo não-regular

Hotéis e similares

Outros tipos de alojamento não especificados anteriormente

Restaurante e outros estabelecimentos de serviços de alimentação e bebidas 
Serviços ambulantes de alimentação

Serviços de catering, bufê e outros serviços de comida preparada

Locação de automóveis sem condutor

Locação de meios de transporte, exceto automóveis, sem condutor

Aluguel de equipamentos recreativos e esportivos

Agências de viagens

Operadores turísticos

Serviços de reservas e outros serviços de turismo não especificado anteriormente

Atividades de museus e de exploração, restauração artística e conservação de lugares e prédios históricos e atrações similares

Atividades de jardins botânicos, zoológicos, parques nacionais, reservas ecológicas e áreas de proteção ambiental

Parques de diversão e parques temáticos

Atividades de recreação e lazer não especificados anteriormente

Fonte: Ribeiro e Andrade (2015)

Esses autores, na ausência de uma Conta Satélite, basearam-se na pesquisa sobre Economia do Turismo conduzida pelo Instituto Brasileiro de Geografia e Estatística (IBGE). De forma resumida, esta pesquisa indica as seguintes atividades pertencentes ao turismo: i) Serviços de alojamento; ii) Serviços de provisão de alimentação e bebidas; iii) Serviços de transporte de passageiros; iv) Serviços de agências de viagens, operadoras e guias de turismo; v.) Serviços culturais; vi) Serviços recreativos e outros serviços de en- tretenimento; e vii) Serviços turísticos diversos.

\section{RESULTADOS E DISCUSSÕES}

No intuito de realizar uma análise exploratória da base de dados, a Tabela 2 apresenta estatísticas descritivas das cinco variáveis que foram utilizadas na análise de cluster. Vale lembrar que a base de dados é constituída de 5.565 observações (municípios brasileiros).

Tabela 2 - Estatísticas Descritivas

\begin{tabular}{lcccc}
\hline Variáveis & Média & $\begin{array}{c}\text { Desvio } \\
\text { Padrão }\end{array}$ & Mínimo & Máximo \\
\hline QL & 0,41 & 0,65 & 0,00 & 11,93 \\
Est_h & 99,17 & 141,37 & 0,00 & 4.881 \\
Renda & 190,35 & 248,90 & 0,00 & $3.360,43$ \\
Edu & 0,17 & 0,09 & 0,00 & 0,97 \\
E_t & 0,03 & 0,04 & 0,00 & 0,74 \\
\hline
\end{tabular}

Fonte: Elaboração Própria

De forma geral, todas as variáveis retratam um grau relativamente forte de heterogeneidade dos dados, uma vez que os respectivos desvios-padrão são altos. A participação do emprego no turismo em relação ao total (E_t) e o número de estabelecimentos turísticos por cada 100.000 habitantes (Est_h), por exemplo, varia de $0 \%$ a $74 \%$ e 0 a 4.481 , respectivamente. A remuneração média do setor de turismo entre os municí- 
pios brasileiros em 2015 foi de apenas $R \$$ 190,35. Além disso, 17\% da mão-de-obra, em média, possuía nível superior completo.

Por meio da utilização do método hierárquico, foi possível gerar o Dendograma representado na Figura 1. A partir dele, foi traçado uma reta horizontal (cor vermelha) no ponto em que se observa maior ramificação da quantidade de grupos, conforme sugestão de Fávero et al. (2009). Neste ponto, a quantidade de linhas verticais que a linha vermelha cruza é a quantidade ideal de clusters, neste caso, seis.

Figura 1 - Dendograma

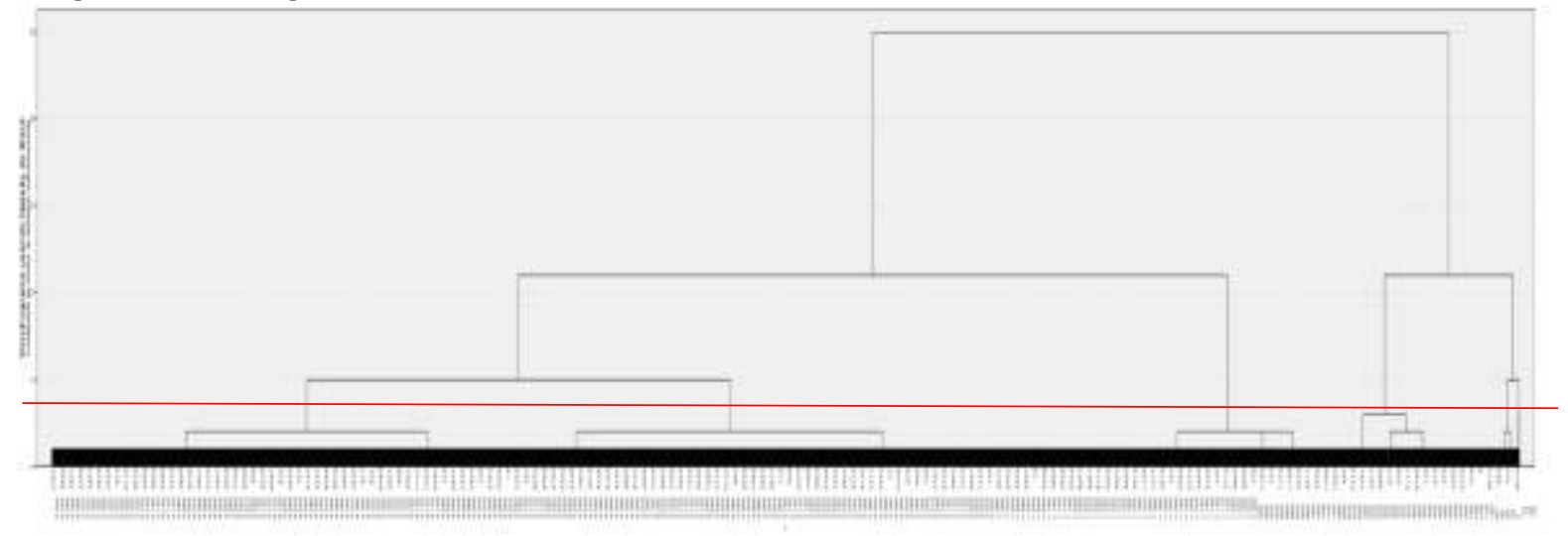

Fonte: Elaboração Própria

Com base nesta informação, utilizouse o método não-hierárquico calibrando-o com seis grupos. A Tabela 3 apresenta a estatística ANOVA, a qual permite verificar qual das variáveis utilizadas no presente trabalho teve maior peso na formação dos clusters, ou seja, a que mais discriminou os grupos.

Tabela 3 - ANOVA

\begin{tabular}{c|c|c|c|c|c|c}
\hline \multirow{2}{*}{ Variável } & \multicolumn{2}{|c|}{ Cluster } & \multicolumn{2}{c|}{ Error } & \multirow{2}{*}{ S } & \multirow{2}{*}{ Sig. } \\
\cline { 2 - 5 } & $\begin{array}{c}\text { Mean } \\
\text { Square }\end{array}$ & df & $\begin{array}{c}\text { Mean } \\
\text { Square }\end{array}$ & Df & \\
\hline QL & 900,86 & 5.00 &, 191 & 5558 & $4.726,84$ & $<0,001$ \\
Est_h & 749,99 & 5.00 &, 326 & 5558 & $2.298,46$ & $<0,001$ \\
Renda & 769,77 & 5.00 &, 308 & 5558 & 2.495 .98 & $<0,001$ \\
Edu & 142,47 & 5.00 &, 873 & 5558 & 163,24 & $<0,001$ \\
E_t & 899,64 & 5.00 &, 192 & 5558 & $4.693,35$ & $<0,001$ \\
\hline
\end{tabular}

Fonte: Elaboração própria a partir do software SPSS

Todas as variáveis utilizadas foram significativas, uma vez que a última coluna (Sig.) apresentou valores $<0,001$ para todas elas. A partir da estatística $F$ é possível observar qual a variável que mais contribuiu para a formação dos clusters. Percebe-se que o QL apresentou maior valor da estatística $\mathrm{F}$ e, portanto, foi ele que mais discriminou a formação dos grupos. Importante destacar que esta variável busca identificar os municípios brasileiros mais especializados no turismo. Os resultados da estimação dos clusters são sintetizados na Tabela 4. 
Tabela 4 - Resultado dos Clusters dos Serviços Turísticos

\begin{tabular}{|c|c|c|c|c|c|c|}
\hline Variável & Cluster & № Municípios & Média & $\begin{array}{l}\text { Desvio Pa- } \\
\text { drão }\end{array}$ & Mínimo & Máximo \\
\hline \multirow{7}{*}{$\begin{array}{l}\text { Quociente locacional } \\
\text { do turismo (QL) }\end{array}$} & 1 & 17 & 6,34 & 1,69 & 4,31 & 10,22 \\
\hline & 2 & 3.230 & 0,12 & 0,14 & 0,00 & 1,14 \\
\hline & 3 & 1.812 & 0,57 & 0,31 & 0,04 & 1,82 \\
\hline & 4 & 139 & 2,68 & 0,92 & 0,14 & 6,7 \\
\hline & 5 & 366 & 1,06 & 0,35 & 0,05 & 1,05 \\
\hline & 6 & 1 & 11,93 & $\ldots$ & 11,93 & 11,93 \\
\hline & Total & 5.565 & 0,41 & 0,65 & 0,00 & 11,93 \\
\hline \multirow{7}{*}{$\begin{array}{l}\text { Número de estabele- } \\
\text { cimento do setor tu- } \\
\text { rístico para cada } \\
100.000 \text { habitantes } \\
\text { (Est_h) }\end{array}$} & 1 & 17 & $1.277,88$ & 643,89 & 397 & 2.837 \\
\hline & 2 & 3.230 & 35,5 & 39,5 & 0,00 & 235 \\
\hline & 3 & 1.812 & 155,94 & 88,77 & 6 & 1.295 \\
\hline & 4 & 139 & 292,39 & 220,31 & 19 & $1.288,37$ \\
\hline & 5 & 366 & 208,30 & 99,32 & 33 & 1.343 \\
\hline & 6 & 1 & 4.881 & $\ldots$ & 4.881 & 4.881 \\
\hline & Total & 5.565 & 97,17 & 141,37 & 0,00 & 4.881 \\
\hline \multirow{7}{*}{$\begin{array}{l}\text { Renda média das ati- } \\
\text { vidades turísticas }\end{array}$} & 1 & 17 & 593,87 & 279,41 & 138,17 & $1.066,93$ \\
\hline & 2 & 3.230 & 58,90 & 61,75 & 0,00 & 470,89 \\
\hline & 3 & 1.812 & 273,24 & 142,39 & 30,44 & 706,39 \\
\hline & 4 & 139 & 349,53 & 255,30 & 42,85 & $1.288,37$ \\
\hline & 5 & 366 & 858,86 & 358,54 & 343,48 & $3.360,43$ \\
\hline & 6 & 1 & 777,12 & $\ldots$ & 777,12 & 777,12 \\
\hline & Total & 5.565 & 190,35 & 248,90 & 0,00 & $3.360,43$ \\
\hline \multirow{7}{*}{$\begin{array}{l}\text { Percentual de traba- } \\
\text { lhadores atuando em } \\
\text { atividades turísticas } \\
\text { com o ensino superior } \\
\text { completo (Edu) }\end{array}$} & 1 & 17 & 0,12 & 0,03 & 0,07 & 0,2 \\
\hline & 2 & 3.230 & 0,19 & 0,10 & 0,00 & 0,97 \\
\hline & 3 & 1.812 & 0,12 & 0,04 & 0,00 & 0,44 \\
\hline & 4 & 139 & 0,13 & 0,07 & 0,02 & 0,77 \\
\hline & 5 & 366 & 0,15 & 0,05 & 0,06 & 0,4 \\
\hline & 6 & 1 & 0,9 & $\ldots$ & 0,9 & 0,9 \\
\hline & Total & 5.565 & 0,17 & 0,09 & 0,00 & 0,97 \\
\hline \multirow{7}{*}{$\begin{array}{l}\text { Número de emprega- } \\
\text { dos do setor turístico } \\
\text { (E_t) }\end{array}$} & 1 & 17 & 0,39 & 0,10 & 0,27 & 0,63 \\
\hline & 2 & 3.230 & 0,01 & 0,008 & 0,00 & 0,07 \\
\hline & 3 & 1.812 & 0,03 & 0,02 & 0,00 & 0,11 \\
\hline & 4 & 139 & 0,16 & 0,06 & 0,01 & 0,44 \\
\hline & 5 & 366 & 0,06 & 0,02 & 0,00 & 0,12 \\
\hline & 6 & 1 & 0,74 & $\ldots$ & 0,74 & 0,74 \\
\hline & Total & 5.565 & 0,02 & 0,04 & 0,00 & 0,74 \\
\hline
\end{tabular}

Fonte: Elaboração própria

A Tabela 4 mostra as principais esta- em que as que se destacam têm forte apelo tísticas descritivas dos seis grupos formados. turístico, principalmente referente ao segNo cluster 1 ficaram agrupados 17 cidades, mento de sol e praia, são elas: Porto Seguro/ 
BA, Mata de São João/BA, Jijoca de Jericoacoara/CE, Bonito/MS, Alto Paraíso de Goiás/GO, Armação de Búzios/RJ, Paraty/RJ, Ilhabela/SP e Gramado/RS. Ao observar o nível de especialização da atividade turística, verificou-se que este grupo apresenta elevado nível de especialização $(Q L=6,34)$, sendo inferior somente ao cluster 6 (QL= 11,93). Essas cidades possuem em média, 1.277 estabelecimentos turísticos para cada 100.00 habitantes; $39 \%$ da mão de obra formal atuam no setor turístico, $12 \%$ dela possuem ensino superior completo e a remuneração média do setor é $\mathrm{R} \$ 593,87$. Dada a característica desse grupamento, o mesmo pode ser considerado como um cluster turístico.

A maioria dos municípios brasileiros, 3.230 (58\%) compõe o cluster 2 . De forma geral, percebe-se que a oferta da atividade turística é incipiente nesses municípios uma vez que, em média, apenas $1 \%$ dos trabalhadores formais atua no setor com remuneração média de $\mathrm{R} \$ 58,90$ e só existem 35,5 estabelecimentos turísticos para cada 100.000 habitantes. Além disso, o QL foi próximo de zero $(0,12)$, indicando que esses municípios não são especializados no segmento turístico. Este resultado sugere que a grande maioria dos municípios brasileiros apresenta estrutura de mão de obra e oferta turística incipiente. Resultado similar foi obtido por Ribeiro e Lopes (2015) para o setor cultural, ou seja, este setor é inexistente na maioria das cidades brasileiras.

O cluster 3 , formado por 1.812 municípios, juntamente com o cluster 1 , apresentou o menor percentual de trabalhadores do setor turístico com o ensino superior com- pleto (12\%). Existem 155 estabelecimentos turísticos para cada 100.000 habitantes, valor bem abaixo dos 1.277 observados no primeiro cluster. Apenas 3\% dos trabalhadores formais estão empregados em atividades associadas ao turismo. Ademais, o rendimento médio de um trabalhador do setor turístico $(R \$ 273,24)$ é cerca de quatro vezes maior do que no cluster 2 . Similarmente ao agrupamento 2, as características deste grupo mostram uma estrutura incipiente do setor turístico.

O cluster 4 foi composto por 139 municípios. De acordo com os resultados, o mesmo poderia ser classificado como um cluster especializado em turismo, uma vez que a média do $Q L=2,68$. Dentre as cidades que formaram esse cluster destacam-se: Barra de São Miguel/AL; Maragogi/AL; São Miguel dos Milagres/AL; Lençois/BA; Vera Cruz/BA; Itacaré/BA; Santa Cruz de Cabralia/BA; Lagoa Santa/GO; Chapada dos Guimarães/MT; Arraial do Cabo/RJ; São Miguel do Gostoso/RN; Balneário Camboriu/SC; Gravatal/SC e Santo Antônio do Pinal/SP, municípios com potencial turístico seja no segmento de sol e praia, no turismo de aventura ou no turismo cultural. Esse grupo apresentou, em média, 292 estabelecimentos turísticos para cada 100.000 habitantes; do total de trabalhadores formais atuando nessas cidades, apenas $16 \%$ estão atuando em atividades turísticas, $13 \%$ tem o ensino superior completo e a renda média é de $\mathrm{R} \$ 349,53$.

Apesar dos grupos 1 e 4 poderem ser considerados cluster turísticos, eles possuem uma renda média muito baixa. Isto pode estar associado, em parte, a baixa qualificação da mão-de-obra, uma vez que o setor só pos- 
sui $12 \%$ e $13 \%$, respectivamente, dos trabaIhadores com ensino superior completo.

O cluster 5 é formado por 366 cidades, dentre as quais se encontram todas as 27 capitais brasileiras. Esses municípios possuem, em média, 208 estabelecimentos turísticos para cada 100.000 habitantes, 6\% dos trabalhadores formais estão no setor turístico, dos quais $15 \%$ têm ensino superior completo. Este grupo apresentou a maior remuneração média do setor turístico ( $R$ \$ 858,86). No entanto, Guimarães e Silva (2013) identificaram uma desigualdade salarial de gênero neste setor, uma vez que $67,79 \%$ dos melhores salários são de homens. É importante ressaltar que quanto maior o tamanho da cidade maior é o grau de diversificação da sua economia. Não obstante, é de se esperar que, ainda que este cluster não seja especializado no turismo, o setor apresenta a maior remuneração média e, relativamente, o terceiro maior nível de escolaridade. Isto é esperado, uma vez que o custo de vida nas cidades grandes e a maior competitividade do mercado de trabalho exigem isso.

Percebe-se que o cluster 6 foi formado somente por Fernando de Noronha/PE, cidade brasileira mais especializada em turismo no ano de 2015 (QL= 11,93). A principal justificativa estatística deste resultado decorre do QL uma vez que, como destacado anteriormente, essa variável foi a que mais discriminou a formação dos grupos. A mesma evidência foi encontrada por Ribeiro et al. (2017) que também identificaram o setor turístico deste município como o mais especializado do Brasil. Observa-se que 74\% dos trabalhadores formais atuavam no setor turístico, dos quais $90 \%$ tinham o ensino su- perior completo e receberam rendimento médio de $\mathrm{R} \$ 777,12$ (segunda maior remuneração entre os grupos). Vale ressaltar que esta cidade representa um dos principais atrativos turísticos do Brasil tanto do ponto de vista nacional quanto internacional. Por outro lado, este resultado deve ser relativizado, uma vez que Fernando de Noronha é uma ilha cuja única atividade econômica é o turismo. Desse modo, o município é totalmente dependente desta atividade e, portanto, como todo o mercado de trabalho gira em torno do turismo, é de se esperar que o município seja especializado no setor.

Ao contrário do que aconteceu neste artigo, nos trabalhos de Machado et al. (2013) e Ribeiro et al. (2014), os quais analisam as indústrias criativa e do lazer, respectivamente, as cidades de São Paulo e Rio de Janeiro ficaram isoladas em um único cluster. Ribeiro e Lopes (2015), analisando o setor cultural no Brasil, por sua vez, identificaram que os municípios Rio de Janeiro e São Paulo ficaram cada um em um cluster separado, formado somente por eles.

De forma geral, pode-se considerar que os grupos 1, 4 e 6 são clusters turísticos, uma vez que os mesmos possuem diversas cidades especializadas no setor. Nos três grupos há municípios com forte potencial turístico em diferentes segmentos. Entretanto, não houve predominância, nesses agrupamentos, de municípios localizados nas regiões mais ricas do país, Sul e Sudeste. Ao contrário do que se verificou no trabalho de Ribeiro e Andrade (2015), que ao analisarem o mercado de trabalho turístico das mesorregiões brasileiras, observaram que o cluster com os melhores resultados foi justamente aquele em que havia predominância de me- 
sorregiões pertencente ao Sul e Sudeste do país. Este resultado é muito importante, pois sugere que o turismo pode ser utilizado como estratégia de desenvolvimento em cidades localizadas em regiões mais pobres, como já discutido por Ribeiro et al. (2017b).

A partir da Figura 2 é possível visualizar a distribuição espacial dos clusters no território brasileiro. Verifica-se que os resultados relativamente piores, dentre as variáveis analisadas, são trazidos pelos clusters 2 e 3 , os quais são constituídos, em sua maioria, por municípios localizados no Norte e Nordeste, regiões mais pobres do país. Portanto, pode-se inferir que em 5.042 municípios brasileiros, o que representa $90,60 \%$ do total, o mercado de trabalho formal do turismo é incipiente. Em outras palavras, a oferta turística mais desenvolvida e estruturada é concentrada em poucos municípios e, não necessariamente, os mesmos pertencem às regiões brasileiras mais ricas.

Figura 2- Distribuição espacial dos clusters - Serviços turísticos

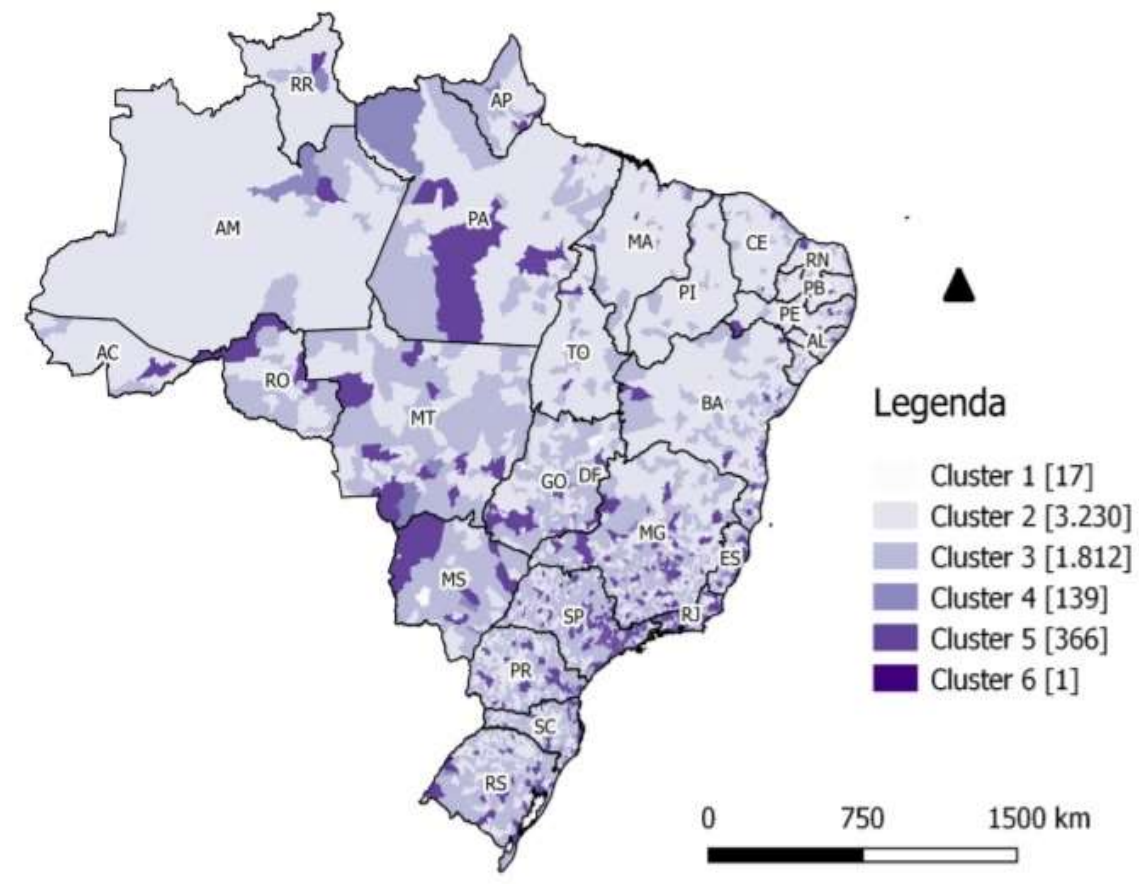

Fonte: Elaboração Própria

\section{CONSIDERAÇÕES FINAIS}

Este trabalho analisou o setor turístico nos municípios brasileiros em 2015. Para isso, utilizou-se a análise de cluster com a finalidade de agrupar os municípios segundo as similaridades dos seus serviços turísticos.

Ao longo do trabalho buscou-se responder à seguinte pergunta de pesquisa: será que o setor de turismo é mais desenvolvido e especializado nas regiões mais ricas do país? A partir da análise conduzida neste artigo pode-se constatar que o cluster mais especializado em turismo foi formado por uma única cidade, Fernando de Noronha/PE, localizada no Nordeste brasileiro. Entretanto, em Fernando de Noronha/PE a atividade econômica predominante é o turismo, deste modo, 
já é esperado que esta localidade apresentasse um alto nível de especialização do setor. Não se pode afirmar que o setor turístico brasileiro é mais especializado e desenvolvido nas regiões mais ricas do país, uma vez que, depois do cluster formado por Fernando de Noronha/PE, o agrupamento 2 foi o que apresentou os melhores resultados e neste grupo não houve predominância de munícipios do Sul e Sudeste do país. A maioria dos municípios brasileiros $(90,60 \%)$ apresenta uma estrutura de oferta e mão de obra turística incipiente (Figura 2).

Diferentemente de estudos anteriores que analisaram a indústria do lazer ( $\mathrm{Ri}$ beiro et al., 2014), a indústria criativa (Machado et al., 2013) e o setor cultural (Ribeiro e Lopes, 2015), em que os municípios de São Paulo e Rio de Janeiro se destacaram em relação as demais cidades brasileiras, os resultados voltados especificamente para as atividades turísticas trás como destaque o município de Fernando de Noronha/PE, o que não é surpresa uma vez que se refere a um dos principais destinos turísticos no Brasil.

A partir dos resultados é possível inferir que os clusters 1, 4 e 6, podem ser classificados como clusters turísticos, uma vez que nestes agrupamentos há uma predominância de cidades com forte apelo turístico, além de terem sido os grupos que apresentaram o maior nível de especialização do setor. No entanto, percebe-se que, de forma geral, há uma baixa qualificação da mão-de-obra que atua no setor (apenas 17\% em média possui nível superior). Com efeito, as políticas de turismo poderiam ter como um dos eixos de atuação programas de qualificação continuada da mão-de-obra.
Os resultados indicaram que o Nordeste, por exemplo, possui diversas cidades com potencial turístico. Desse modo, tais cidades podem utilizar o turismo como estratégia de desenvolvimento. Para tanto, primeiramente, é necessária a estruturação do setor como, por exemplo, infraestrutura turística, divulgação do destino, entre outras ações.

A principal limitação do presente artigo é a utilização de dados formais, o que impossibilita resultados mais específicos de acordo com a real situação do setor turístico brasileiro. Todavia, como já mencionado anteriormente, não existem dados informais atuais do mercado de trabalho turístico a nível municipal. Os dados mais recentes têm como ano base 2010, ou seja, também não retratariam a situação atual do turismo no Brasil. Diante de tais dificuldades, optou-se pela utilização de dados mais atuais, mas sem levar em consideração a informalidade.

Diante do exposto e da importância da atividade turística na geração de desenvolvimento local/regional, seja de maneira direta ou indireta, é importante a atuação do poder público por meio da criação de políticas eficientes com a finalidade de promover o desenvolvimento harmônico do setor, maximizando os impactos positivos provocados por tal atividade e minimizando seus efeitos negativos.

Associado a isso, é de fundamental importância o esforço contínuo do setor público no sentido de produzir novas informações estatísticas sobre o setor de turismo no Brasil. Neste quesito, parcerias público-privadas podem aparecer como uma boa estratégia. Desse modo, isto pode fomentar novas 
pesquisas e, consequentemente, diagnósticos e direcionamentos mais eficientes.

\section{REFERÊNCIAS}

Ablas, L. (1991). Efeitos do turismo no desenvolvimento regional. Revista Turismo em Análise, 2 (1), 42-52.

Anato, M. (2006). El uso de los instrumentos clásicos del marketing y la tecnología digital en turismo. Estudios y Perspectivas em Turismo, 15, 11-44.

Aulicino, M. P. (2011). Turismo e desenvolvimento regional: um estudo no estado de São Paulo. Revista Turismo em Análise, 22 (1), 220234.

Barbosa, F. F. (2005). Turismo como um fator de desenvolvimento local e/ou regional. Caminhos de Geografia, 6 (14), 107-114.

Beni, M. C. (2003). Globalização do Turismo: megatendência do setor e a realidade brasileira. Aleph: São Paulo.

Brasil. Ministério do Turismo. (2007). Turismo no Brasil 2007-2010. Brasília/DF: MTUR.

Brasil. (2010). Programa de regionalização do turismo - Roteiros do Brasil: módulo operacional 7-Roteirização Turística. Brasília, DF.

Combes, P. P. (2000). Economic structure and local growth: France, 1984-1993. Journal of Urban Economics , 47(3), 329-55.

Fávero L. P., Belfiore, P., Silva, F. L., \& Chan, B. L. (2009). Análise de dados: modelagem multivariada para tomada de decisões. Rio de Janeiro: Elsevier.

Garsous, G., Corderi, D., Velasco, M., \& Colombo, A. (2017). Tax incentives and job creation in the tourism sector of Brazil's SUDENE area. World Development. Doi: http://dx.doi.org/10.1016/j.worlddev.2017.02.0 34.
Glaeser, E. L., Kallal, H. D., Scheinkman, J. A., \& Shleifer, A. (1992) Growth in cities. Journal of Political Economy, 100(6), 1126-1152.

Guimarães, C. R. F. F., \& Silva, J. R. (2016). Pay gap by in the tourism industry of Brazil. Tourism Management, 52, 440-450.

Haddad E. A., Porsse, A. A., \& Rabahy, W. A. (2013). Domestic tourism and regional inequality in Brazil. Tourism Economics 19(1), 173-186.

Haddad, P. R. (1989). Economia regional, teorias e métodos de análise. Fortaleza: BNB/ ETENE. Hair Jr, F.F., Anderson, R. E., Tathan, R. L., \& Black, W. C. (2005). Análise multivariada de dados. Bookmas,, Porto Alegre.

Hurtado, J. M. R., \& Bonilla, J. M. L. (2012). Classificación de las zonas turísticas españolas en función de las características estructurales de la oferta y la demanda - una aplicación mediante un modelo de classes latentes. Estudios y Perspectivas en Turismo, 21 (1), 34-51.

IPEA. Instituto de Pesquisa Econômica Aplicada. (2015). Relatório com as estimativas da caracterização da ocupação formal e informal do turismo, com base nos dados da RAIS e da PNAD 2013, para o Brasil e regiões. Disponível em: http://www.ipea.gov.br/extrator/arquivos/160204_caracterizacao_br_re.pdf, acesso em 14 de janeiro de 2018.

Lage, B. H. G., \& Milone, P. C. (1991). Economia do Turismo. Campinas-SP, Papirus.

Lazzaretti, L., \& Capone, F. (2009). Spatial spillovers and employment dynamics in Local Tourist Systems in Italy (1991-2001). European Planning Studies, 17(11), 1665-1683.

Linden, R. (2009). Técnicas de agrupamentos. Revista de Sistemas de Informação da FSMA, 4: 18:31.

Lins, H. N. (2000). Florianópolis, cluster turístico?. Turismo em Análise, 11 (2): 55-70. 
Machado, A. F., Simões, R., \& Diniz, S. C. (2013). Urban amenities and the development of creative clusters: the case of Brazil. Current Urban Studies, 1, 92-101.

Malhotra, N. (2006). Pesquisa de markenting: uma orientação aplicada. Trad. Laura Bocco. $4^{\circ}$ ed. Porto Alegre: Bookman.

Massardi, W. O., Abrantes, L. A. (2014). Classificação dos municípios mineiros em relação a composição de suas receitas. Revista de Gestão, Finanças e Contabilidade, 4 (1), 144-161.

Moreira Filho, M., Nascimento, R. P., \& Segre, L. M. (2010). Cual es el papel del turismo en el desarollo local? Un análisis crítico del cluster turístico de Santa Tereza - RJ, Brasil. Estudios y Perspectivas en Turismo, 19 (5), 812-834.

Porter, M. E. (1998). Clusters and the new economics of competition. Harvad Business Review,76 (6), 77-90.

Ribeiro, L. C. S., \& Andrade, J. R. L. (2015). Characterization of tourism clusters in Brazil. Tourism Economics, 21, 957-976.

Ribeiro, L. C. S., Andrade, J. R. L., \& Pereira, R. M. (2013). Estimação dos benefícios econômicos do Prodetur Nacional em Sergipe. Revista Economia Nordeste, 44 (4), 975-1000.

Ribeiro, L. C. S., \& Lopes, T. H. C. R. (2015). Características e similaridades do setor cultural nos munícipios e regiões metropolitanas brasileiras. Revista de Economia Contemporânea, 19 (2), 307-330.

Ribeiro, L. C. S., Lopes, T. H. C. R., Montenegro, R. L., \& Andrade, J. R. L. (2017a). Employment dynamics in the Brazilian tourism sector (20062015). Tourism Economics. Doi: https://doi.org/10.1177/1354816617736409.
Ribeiro, L. C. S., Nahas, M., Amaral, P. V., \& Simões, R. (2014). A indústria do lazer no Brasil e sua relação com o desenvolvimento municipal. Revista Brasileira de Estudos Regionais e Urbanos, 8(1), 77-91.

Ribeiro, L. C. S., Silva, E. O. V.. Andrade, J. R. L., \& Souza, K. B. (2017b). Tourism and regional development in the Brazilian Northeast. Tourism Economics, 23(3), 717-727.

Tomé, L. M. (2017). Turismo no Nordeste: aspectos gerais. Caderno Setorial Etene, Ano $2, \mathrm{~N}^{\circ}$ 20.

World Travel and Tourism Council (WTTC). (2016). Travel and tourism economic impact 2016. Disponível em: https://www.wttc.org//media/files/reports/economic\%20impact\%20research/regions\%202016/\%20world2016.pdf. Acesso em 05 de dezembro de 2017.

Informations on the authors

Fernanda Rodrigues dos Santos

Graduada em Economia, Universidade Federal de Sergipe. E-mail: fxnanda@hotmail.com ORCID: 0000-0002-8998-6720.

\section{Luiz Carlos de Santana Ribeiro}

Professor Assistente do Departamento de Economia, Universidade Federal de Sergipe. Bolsista do CNPq e Coordenador do Laboratório de Economia Aplicada e Desenvolvimento Regional- LEADER.

E-mail: ribeiro.luiz84@gmail.com

ORCID: 0000-0001-6374-3811.

\section{Emanuel Jhonata Gomes da Silveira}

Mestrando em Economia, Universidade Federal de Sergipe e pesquisador do LEADER. E-mail: emanueljhon@hotmail.com

ORCID: 0000-0003-2837-0970. 
Anexo 1A - Percentual de informalidade das (ACTs) por região (\%)

\begin{tabular}{|c|c|c|c|c|}
\hline Região & ACTs & 2012 & 2013 & 2014 \\
\hline \multirow{8}{*}{ Norte } & Alojamento & 31 & 27 & 32 \\
\hline & Alimentação & 80 & 79 & 76 \\
\hline & Transporte Terrestre & 61 & 60 & 59 \\
\hline & Transporte Aquaviário & 53 & 53 & 51 \\
\hline & Transporte Aéreo & 12 & 5 & 9 \\
\hline & Aluguel de Transportes & 62 & 49 & 50 \\
\hline & Agência de Viagem & 35 & 44 & 44 \\
\hline & Cultura e Lazer & 74 & 73 & 66 \\
\hline \multirow{8}{*}{ Nordeste } & Alojamento & 26 & 23 & 23 \\
\hline & Alimentação & 73 & 72 & 72 \\
\hline & Transporte Terrestre & 68 & 67 & 66 \\
\hline & Transporte Aquaviário & 33 & 28 & 15 \\
\hline & Transporte Aéreo & 4 & 5 & 7 \\
\hline & Aluguel de Transportes & 36 & 39 & 47 \\
\hline & Agência de Viagem & 47 & 48 & 50 \\
\hline & Cultura e Lazer & 77 & 73 & 72 \\
\hline \multirow{8}{*}{ Sudeste } & Alojamento & 18 & 16 & 18 \\
\hline & Alimentação & 51 & 51 & 51 \\
\hline & Transporte Terrestre & 42 & 40 & 39 \\
\hline & Transporte Aquaviário & 23 & 25 & 25 \\
\hline & Transporte Aéreo & 3 & 4 & 6 \\
\hline & Aluguel de Transportes & 21 & 15 & 16 \\
\hline & Agência de Viagem & 33 & 34 & 31 \\
\hline & Cultura e Lazer & 62 & 62 & 63 \\
\hline \multirow{8}{*}{ Centro-Oeste } & Alojamento & 25 & 21 & 20 \\
\hline & Alimentação & 58 & 55 & 55 \\
\hline & Transporte Terrestre & 38 & 39 & 37 \\
\hline & Transporte Aquaviário & 68 & 42 & 69 \\
\hline & Transporte Aéreo & 13 & 11 & 12 \\
\hline & Aluguel de Transportes & 22 & 24 & 37 \\
\hline & Agência de Viagem & 25 & 32 & 34 \\
\hline & Cultura e Lazer & 65 & 63 & 61 \\
\hline \multirow{8}{*}{ Sul } & Alojamento & 23 & 17 & 19 \\
\hline & Alimentação & 50 & 46 & 46 \\
\hline & Transporte Terrestre & 38 & 36 & 34 \\
\hline & Transporte Aquaviário & 15 & 12 & 8 \\
\hline & Transporte Aéreo & 9 & 5 & 5 \\
\hline & Aluguel de Transportes & 10 & 7 & 25 \\
\hline & Agência de Viagem & 59 & 48 & 48 \\
\hline & Cultura e Lazer & 56 & 55 & 55 \\
\hline
\end{tabular}

Fonte: IPEA 
Anexo 1B: Percentual de informalidade por segmento no Brasil (\%) (2012-2014)

\begin{tabular}{|l|c|c|c|}
\hline \multicolumn{1}{|c|}{ ACTs } & $\mathbf{2 0 1 2}$ & $\mathbf{2 0 1 3}$ & $\mathbf{2 0 1 4}$ \\
\hline Alojamento & 22 & 19 & 21 \\
\hline Alimentação & 59 & 58 & 57 \\
\hline Transporte Terrestre & 46 & 44 & 42 \\
\hline Transporte Aquaviário & 46 & 45 & 41 \\
\hline Transporte Aéreo & 5 & 5 & 6 \\
\hline Aluguel de Transportes & 32 & 28 & 36 \\
\hline Agência de Viagem & 41 & 40 & 39 \\
\hline Cultura e Lazer & 66 & 65 & 64 \\
\hline
\end{tabular}

Fonte: IPEA. 